\title{
Negative Refraction and Subwavelength Focusing in Two-Dimensional Photonic Crystals
}

\author{
E. Ozbay and G. Ozkan
}

Summary. We experimentally and theoretically demonstrate negative refraction and focusing on electromagnetic (EM) waves by using two-dimensional photonic crystal slabs at microwave frequencies. Negative refraction is observed both for transverse magnetic (TM) and transverse electric (TE) polarized incident EM waves. Gaussian beam shifting method is used to verify negative refractive index. Subwavelength imaging and flat lens behavior of photonic crystals are successfully demonstrated. We have been able to overcome the diffraction limit and focus the EM waves to a spot size of $0.21 \lambda$. Metallodielectric photonic crystals are employed to increase the range of angles of incidence that results in negative refraction. Experimental results and theoretical calculations are in good agreement throughout the work.

\subsection{Introduction}

Materials which possess negative index of refraction have become a remarkable research area in recent years [1-10]. One approach taken is to construct a composite metamaterial [2-5] consisting of two components which have a negative permittivity [11] $(\varepsilon(\omega)<0)$ and negative permeability [12] $(\mu(\omega)<0)$ simultaneously over a certain frequency range, respectively, so that the resulting index of refraction of the effective medium becomes $n_{\text {eff }}=\sqrt{\varepsilon} \sqrt{\mu}<0$ [6-10]. Such structures are able to behave like a perfect lens, where both the propagating and evanescent waves contribute to the focusing [13]. Another path is revealed by the photonic crystals (PCs), where the band structure can lead to negative dispersion for electromagnetic (EM) waves.

Photonic crystals are periodic dielectric or metallic structures that have photonic bands exhibiting arbitrarily different dispersions for the propagation of EM waves, and band gaps, where the propagation is prohibited at certain range of wavelengths [14-18]. In this respect, there is a close analogy between a photon in a photonic crystal, and an electron in a semiconductor. Based on these properties, photonic crystals provide a medium where the propagation of light can be modified virtually in any way in a controllable 
manner [19-31]. Their application potential covers the existing electromagnetic technologies for improvement, and extends beyond for advancement. From the fundamental physics point of view, photonic crystals provide access to novel and unusual optical properties. It has been theoretically shown that photonic crystals may possess negative refraction although they have a periodically modulated positive permittivity and permeability of unity [32-35]. Cubukcu et al. has been first to demonstrate negative refraction phenomenon in two-dimensional (2D) PCs in the microwave region [36]. Further experimental studies proved that carefully designed PCs are candidates for obtaining negative refraction at microwave [37] and infrared [38] frequency regimes. Superprism effect is another exciting property arising from photonic crystals $[39,40]$. Subwavelength imaging and resolution [41] and flat lens behavior [42] of PCs have been experimentally demonstrated. Extensive numerical [43-46] and experimental studies [47-52] helped to have a better understanding of negative refraction, focusing, and subwavelength imaging in photonic crystal structures.

In this chapter, we review certain recent studies on the negative refraction and imaging of EM waves by photonic crystal slabs in the microwave frequency regime. We first report on the negative refraction and the subwavelength imaging with transverse magnetic (TM) polarized EM waves. Then we show that it is also possible to have negative refraction with transverse electric (TE) polarized EM waves. Fifth band of the photonic crystal is utilized for purpose of achieving negative refraction. A spectral negative refraction and focusing behavior will be provided. Finally we demonstrate a metallodielectric PC that possesses negative refraction and imaging through a slab. Throughout the work two basic mechanisms arising from the band structure of PCs are employed to obtain negative refraction. We found that focusing abilities of a PC slab can surpass that of conventional (i.e., positive refractive) materials, providing both subwavelength imaging and true flat lens behavior.

\subsection{Negative Refraction and Subwavelength Imaging of TM Polarized Electromagnetic Waves}

Refraction is perhaps one of the most basic topics of electromagnetic phenomena, whereby when a beam of radiation is incident on an interface between two media at an arbitrary angle, the direction of propagation of the transmitted beam is altered by an amount related to the indices of refraction of the two media. Although all of the known naturally occurring materials exhibit positive indices of refraction, the possibility of materials with negative refractive index has been explored theoretically by Victor Veselago [1]. In his seminal work, he concluded that such materials did not violate any fundamental physical laws. These materials were termed left-handed materials (LHMs), 
and it was further shown that some of the most fundamental electromagnetic properties of an LHM would be opposite to that of ordinary right-handed materials (RHM), resulting in unusual and nonintuitive optics [5,8]. Photonic crystals are alternatives to LHM structures, being a candidate for negative refractive media. Recent experimental and theoretical works indicate that negative refraction phenomena in photonic crystals are possible. Luo et al. demonstrated theoretically all-angle negative refraction of two-dimensional PCs [34].

The $2 \mathrm{D}$ photonic crystal structure that we use in our experiments consists of a square array of dielectric rods in air having a dielectric constant $\varepsilon=9.61$, radius $r=1.6 \mathrm{~mm}$, and length $l=150 \mathrm{~mm}$ [36]. The periodicity of the structure in both directions is $a=4.79 \mathrm{~mm}$. The analysis of Luo et al [34] is followed to determine the negative refraction frequency range of our structure, which is calculated to be $13.10-15.44 \mathrm{GHz}$. Propagation properties of the EM wave within the crystal can be described by studying equal-frequency contours (EFCs) in $k$-space. The TM polarized band diagram of the photonic crystal calculated by plane wave expansion method is shown in Fig. 6.1a. We focus on the first band for the experimental and theoretical demonstration of single-beam negative refraction in $2 \mathrm{D}$ photonic crystals. EFCs of the photonic crystal and air at $13.698 \mathrm{GHz}$ are schematically drawn in Fig. 6.1b. The conservation of the surface-parallel wave vector gives the direction of the refracted waves inside the PC [34]. The negative refraction effect is present at this frequency.

The refraction spectrum is measured by a setup consisting of an HP 8510C network analyzer, a standard high-gain microwave horn antenna as the transmitter, and a monopole antenna as the receiver (Fig. 6.2a). The size of the monopole antenna is $11 \mathrm{~mm}$, which is half of the operation wavelength

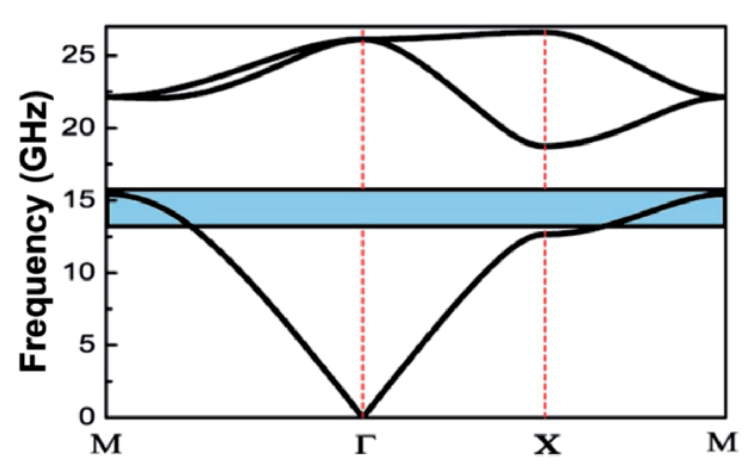

(a)

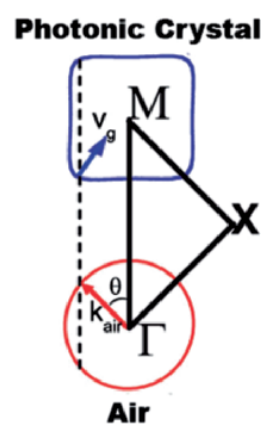

(b)

Fig. 6.1. (a) Calculated band diagram of $2 \mathrm{D}$ photonic crystal for transverse magnetic (TM) polarization. (b) Equal-frequency contours in $k$-space of $\mathrm{PC}$ and air at $13.698 \mathrm{GHz} . \theta$ is the incident angle and $v_{\mathrm{g}}$ is the group velocity inside the $\mathrm{PC}$ 

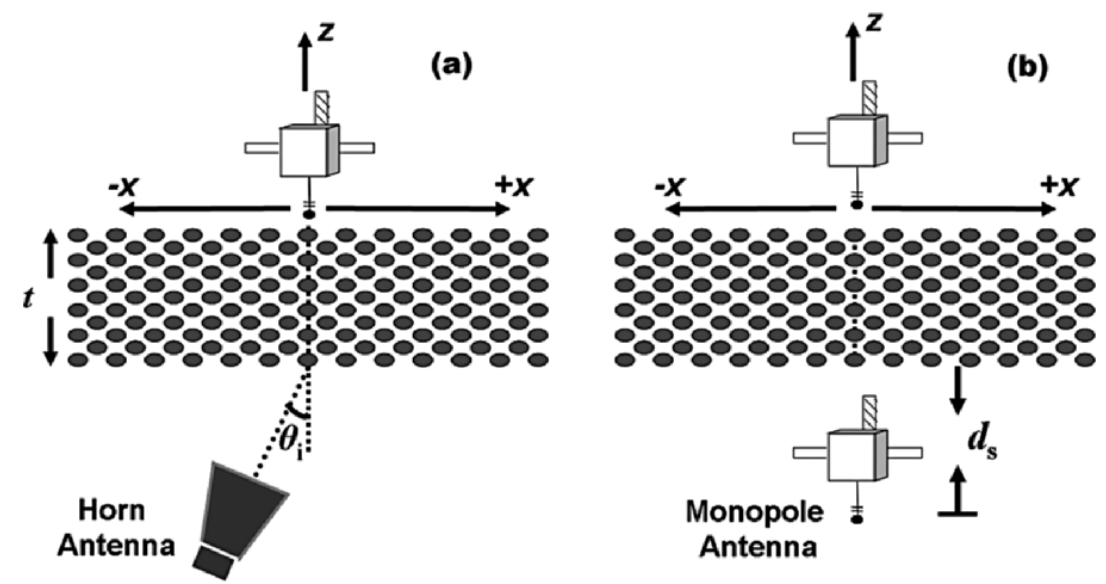

Fig. 6.2. Schematic drawing of the experimental setup for observing (a) negative refraction phenomenon, (b) focusing effect of a slab of negative refractive $2 \mathrm{D}$ PC

$(\lambda \sim 22 \mathrm{~mm})$ of the EM wave at a working frequency of $f=13.698 \mathrm{GHz}$. The top view of the experimental setup is given in Fig. 6.2a. The $x$ and $z$ directions are shown in the figure, whereas the $y$ is directed out of the page. The electric field is along the $y$-direction (i.e., parallel to the rods), whereas the magnetic field and wave-vector are on the $x-z$ plane. The horn antenna is oriented such that the incident waves make an angle of $45^{\circ}$ with the normal of $\Gamma-M$ interface.

The spatial distributions of the time-averaged incident field intensity along the second (PC-air) interface are measured (Fig. 6.3a). The PC used in negative refraction experiments has 17 layers along the propagation direction $(z)$ and 21 layers along the lateral direction $(x)$. For a direct comparison of theoretical predictions and experimental results, simulation of the structure based on experimental parameters using a finite difference time domain (FDTD) method is performed. The incident EM wave has a Gaussian beam profile centered at $x=0$. Therefore, by measuring the shift of the outgoing beam as given in Fig. 6.3a, one can easily deduce whether the structure has a positive or negative refractive index.

Figure 6.3b plots the measured (solid) and simulated (dashed-dotted) spatial distributions of intensity at the interfaces for the slabs of PC (black) and randomly filled polystyrene pellets (gray). As clearly seen in Fig. 6.3b, the center of the outgoing Gaussian beam is shifted to the left side of the center of the incident Gaussian beam for the PC structure. Due to Snell's law, this behavior corresponds to negative refraction. Experimental results and numerical simulations agree well. Refractive index of $\mathrm{PC}$ at $13.698 \mathrm{GHz}$ determined from the experiment is -1.94 , which is very close to the theoretical value of -2.06 computed by the FDTD method. For comparison purposes, the measurements and the simulations are repeated with a slab that contains only polystyrene 


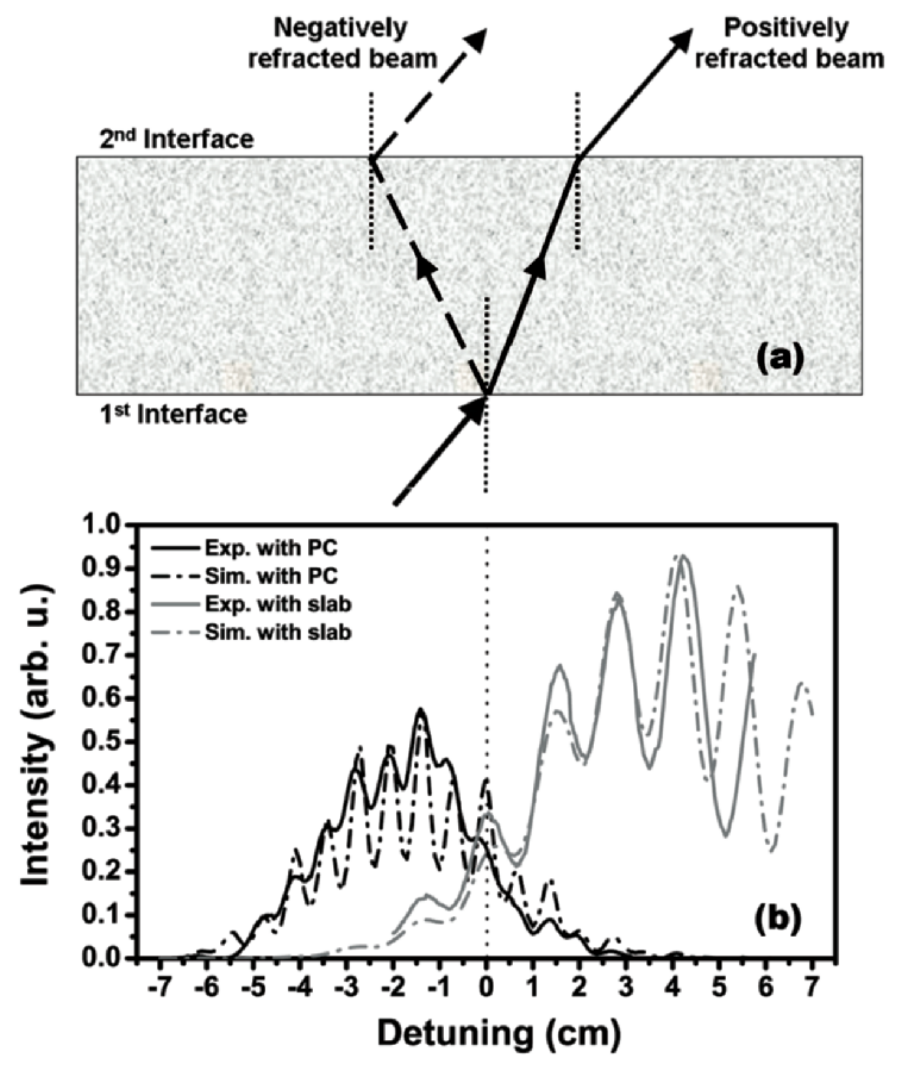

Fig. 6.3. (a) Schematics of refraction of the incident beam through positive and negative media for TM polarization. (b) Refraction spectrum of incident beam through negative refractive PC (black lines) and positive refractive polystyrene pallets (gray lines). The solid lines and the dashed-dotted lines correspond to the experimental measurements and theoretical simulations, respectively

pellets, which has a positive refractive index $(n=1.46)$. The refracted beam emerges from the right-hand side of the incident beam as plotted in Fig. 6.3b. The positive refractive index determined from the experiment is 1.52 , which is close to the tabulated value of 1.46 .

A negative refractive index allows a flat lens to bring EM waves into focus, whereas positive refractive index materials always require curved surfaces to focus EM waves $[1,13]$. One interesting physical behavior of negative index materials is that they can restore the amplitude of evanescent waves and therefore enable subwavelength focusing $[13,43]$. Subwavelength resolution was experimentally verified for negative index materials made of PCs [36]. To investigate the focusing ability of the present PC, a slab of the same PC (with $15 \times 21$ layers) is employed. The operation frequency is set to $13.698 \mathrm{GHz}$, having the largest angular range for negative refraction [36]. FDTD simulations 


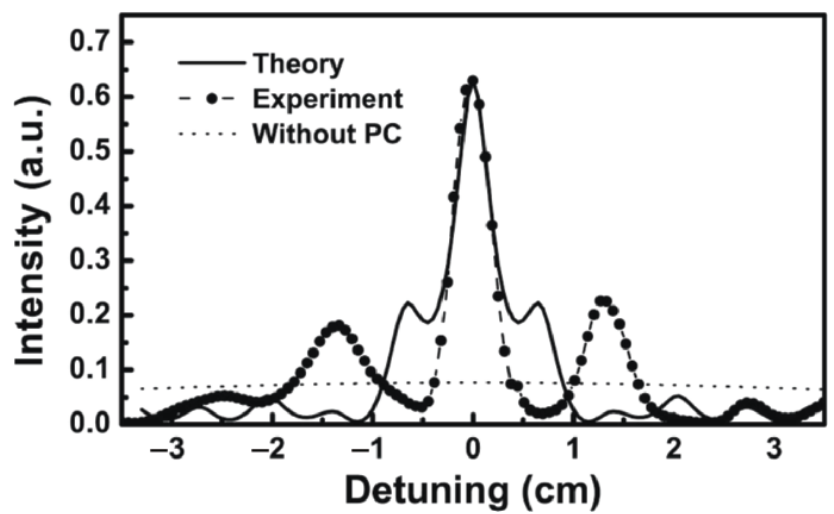

Fig. 6.4. Measured power distribution (large dotted-dashed line) and calculated average intensity (solid line) at the focal point for TM polarization. Dashed line is the spatial power distribution without PC

with experimental parameters predict the formation of an image $0.7 \mathrm{~mm}$ away from the $\mathrm{PC}$-air interface for a point source that is placed $0.7 \mathrm{~mm}$ away from the air-PC interface. We first simulated the distribution of time-averaged intensity along the PC-air interface with and without the PC (solid curve and thin dotted curve in Fig. 6.4). In the experiment, a monopole antenna is used as the point source (Fig. 6.2b). The measured (large dotted-dashed) and calculated (solid) power distribution along the interface is depicted in Fig. 6.4. The full width at half maximum (FWHM) of the measured focused beam is found to be $0.21 \lambda$, which is in good agreement with the calculated FWHM. In contrast, the calculated FWHM of the beam at this plane in the absence of the PC is found to be 5.94 $\lambda$ (dashed line in Fig. 6.4). This implies an enhancement of the transmitted field about 25 times compared to that of free space.

\subsection{Negative Refraction and Point Focusing of TE Polarized Electromagnetic Waves}

In Sect. 6.2 the negative refraction originating from the convex EFCs of the first band around the $M$-point in $k$-space was investigated. The first band has a partial gap around $\Gamma$-point, and the EM waves are forced to move along the $\Gamma-M$-direction, where the conservation of the surface-parallel component of the wave vector causes negative refraction (Fig. 6.1a). In this section, we employ a different band topology of a 2D PC to obtain negative refraction [47]. We aim to achieve negative refractive index with higher isotropy. Based on the analysis presented in [45], we utilize a TE polarized upper band of the PC where the magnetic field is parallel to the dielectric rods. A similar study using the TM polarized band was recently reported by Martinez et al. [49] 

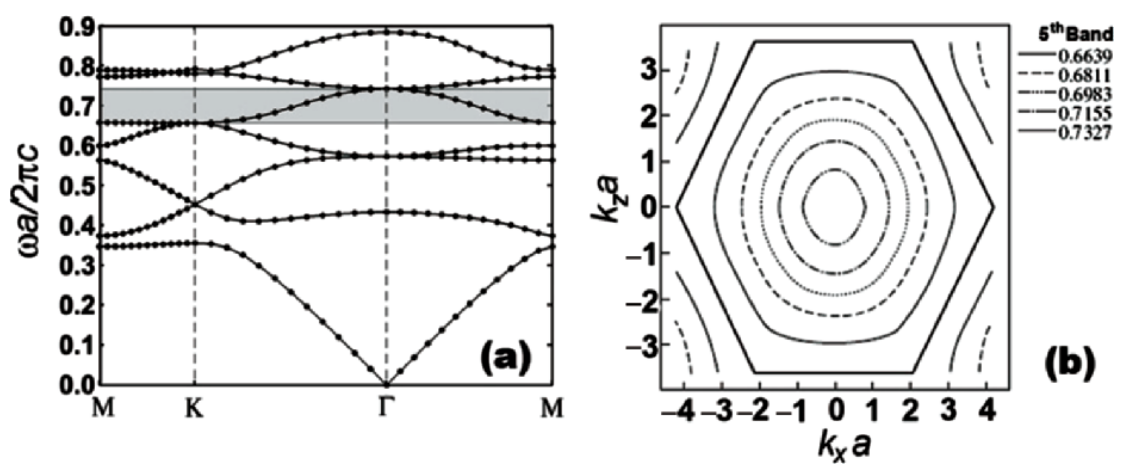

Fig. 6.5. (a) Calculated TE polarized band structure. Shaded band (fifth band) covers the frequencies where the structure possesses negative index of refraction. (b) Equal-frequency contours of the fifth band in the full Brillouin zone. The contours are nearly circular (i.e., isotropic) and shrink with increasing frequency

We used the same photonic crystal structure as analyzed in Sect. 6.2. The only difference is that we calculated the band diagram for TE polarized EM waves. Figure 6.5a depicts the calculated band diagram in the first Brillouin zone. The transverse direction is taken to be in the plane of 2D PC. We scaled frequency with $\tilde{f}=f(a / c)$. The fifth band as shaded in the Fig. 6.5a extends from $\tilde{f}=0.65(f=40.65 \mathrm{GHz})$ to $\tilde{f}=0.74(f=46.27 \mathrm{GHz})$. Figure $6.5 \mathrm{~b}$ plots the EFCs in the full Brillouin zone. The EFCs of the band shrink with increasing frequency, contrary to the EFCs in air $(n=1)$.

The refraction spectra are measured by using same setup as in Fig. 6.2a. Since the EM wave is TE polarized in this case, the horn and the monopole antennas are rotated by $90^{\circ}$. Therefore the magnetic field is parallel to the dielectric rods. The PC structure consists of seven layers along the propagation direction $(z)$ and 31 layers along the lateral direction $(x)$. The horn antenna is on the negative side of the $\mathrm{PC}$ with respect to its central axis. The scanning is performed along the second PC-air interface by $\Delta x=1.27 \mathrm{~mm}$ steps. Refraction spectra of the EM waves with three different incident angles are measured and the results are plotted in Fig. 6.6. The top part of Fig. 6.6 gives the field distribution along the $\mathrm{PC}$-air interface as a function of frequency. It is evident that the refracted beam appears (for $\theta_{\mathrm{i}}=15^{\circ}$ (a), $\theta_{\mathrm{i}}=30^{\circ}$ (b), and $\left.\theta_{\mathrm{i}}=45^{\circ}(\mathrm{c})\right)$ on the negative side, meaning that the PC structure has negative refractive index between 40.0 and $43.0 \mathrm{GHz}$.

When the incidence angle is increased, the transmission shifts to the left accordingly. To investigate the beam profiles, the spatial cross-sections at $f=41.7 \mathrm{GHz}$ are plotted in the middle part of Fig. 6.6. The intensities are normalized with respect to the maximum intensity for the incident angle $\theta_{\mathrm{i}}=15^{\circ}$. It is apparent that the lateral shift is accompanied by a decrease in the transmission intensity. This is due to the higher reflection at the interface for larger incidence angles and the diffraction-induced out-of-plane losses, which increase 

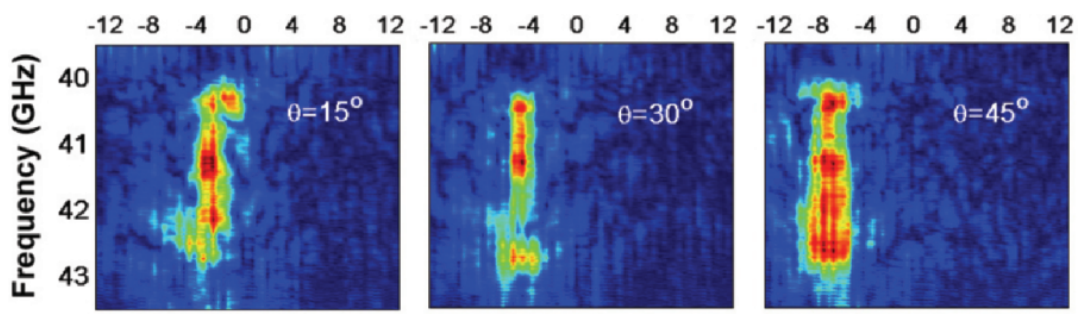

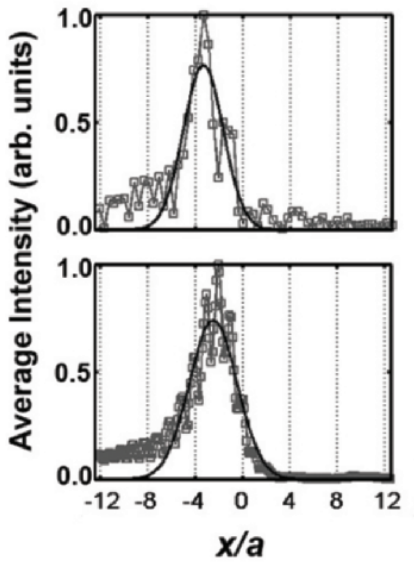

(a)

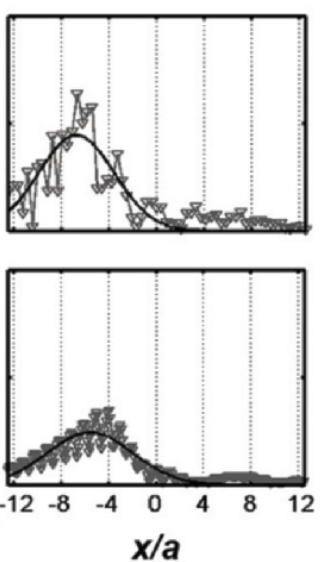

(b)

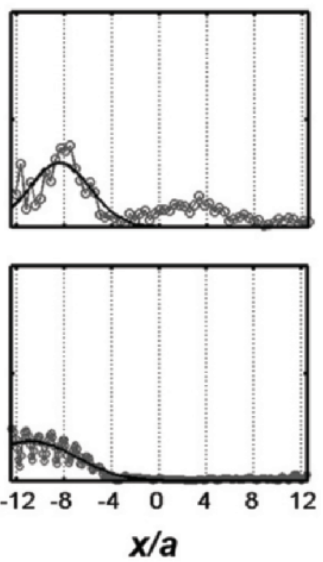

(c)

Fig. 6.6. Measured refraction spectra of the fifth band along the $\mathrm{PC}-$ air interface for incidence angles of (a) $\theta_{\mathrm{i}}=15^{\circ}$, (b) $\theta_{\mathrm{i}}=30^{\circ}$, and (c) $\theta_{\mathrm{i}}=45^{\circ}$ is given on the top figures. Middle figures are the measured, whereas the bottom figures are the simulated intensity profiles at $41.7 \mathrm{GHz}$ for the respective angles of incidence. Solid curves indicate the Gaussian fits of the data. All results are for TE polarization

with increasing path length through the lattice. The bottom part of Fig. 6.6 displays the simulated average field intensity at $f=41.7 \mathrm{GHz}$. Experimental results agree well with the FDTD simulations. By using Snell's law, index of refraction is obtained to be $n_{\text {eff }}=-0.52,-0.66$, and -0.86 from the experiment for $\theta_{\mathrm{i}}=15^{\circ}, 30^{\circ}$, and $45^{\circ}$, respectively. The simulation results for the same incidence angles give $n_{\text {eff }}=-0.66,-0.72$, and -0.80 . The experimental and theoretical results agree quite well. We observed that the band manages to provide a negative refracted uniform beam within the measured frequency range both from the experiments and simulations.

We have also checked the imaging properties of our PC slab for TE polarized EM waves. FDTD simulations are performed at $f=42.07 \mathrm{GHz}$ located at a distance $d_{\mathrm{s}}=2 \lambda$ away from air-PC interface. Omnidirectional (point) source is used to excite the first interface. Figure 6.7a displays the resulting spatial intensity distribution in the image plane, normalized to the value of maximum intensity. The PC-air interface is located at $z=0$. The peak indicates focusing behavior unambiguously. We would like to emphasize that the focusing 

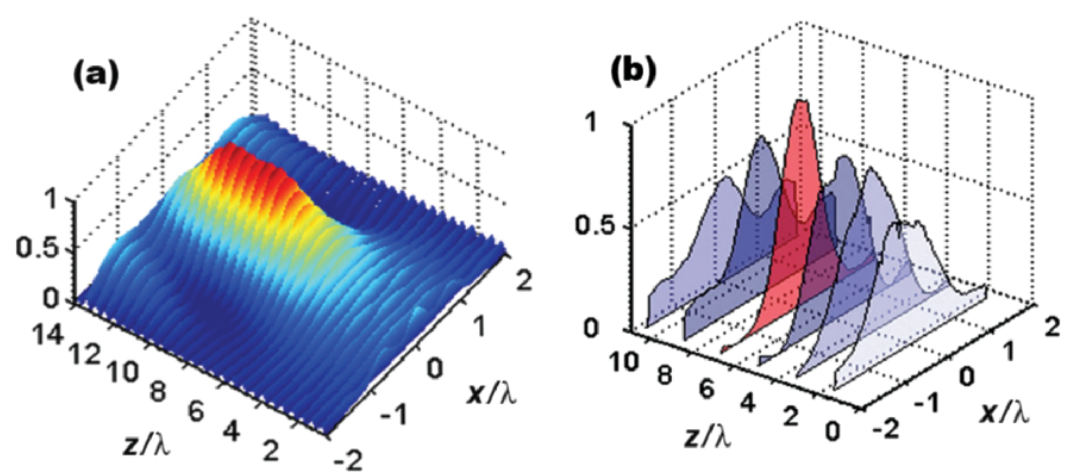

Fig. 6.7. (a) Simulated $2 \mathrm{D}$ intensity profile in the image plane. $z=0$ corresponds to the PC-air interface. (b) Measured lateral intensity profiles along the propagation direction. Measurements are performed at six different positions $z / \lambda=1.78,3.56$, $5.34,7.12,8.90$, and 10.68 for TE polarization

occurs away from the $\mathrm{PC}$-air interface, observed at $z \approx 8 \lambda$. Therefore, unlike the focusing discussed in Sect. 6.2 for TM polarized EM wave, this PC does not perform imaging in the strict sense. Also a subwavelength imaging as presented in Fig. 6.4 is not present since the focal point is quite far away from the interface.

Experimental setup for verifying focusing through a slab of PC is similar to the one discussed in Sect.6.2 (Fig. 6.2b). But to imitate a TE polarized monopole antenna, we employed a waveguide aperture as the source. The waveguide aperture provides sufficiently omnidirectional radiation due to the diffraction at the aperture [47]. The intensity distribution in the focusing plane is measured by the monopole antenna. For $d_{\mathrm{s}}=2 \lambda$, first a scan is performed along the propagation direction $(z)$ to locate the maximum intensity, i.e., the focal point. Then, lateral cross-sections (along $x$ ) of field intensity at several $z$ points around the peak position are measured. As seen in Fig. 6.7b, the beam is focused both in lateral and longitudinal directions. The maximum intensity is observed at $z \approx 8 \lambda$, the focal point. The longitudinal extent of the focusing indicates that index of refraction deviates from negative unity, and bears a certain amount of anisotropy. We stress that even when refractive indices were perfectly isotropic and uniform, a value different from $n=-1$ would not generate point focusing and would induce an aberration of the image.

\subsection{Negative Refraction and Focusing Analysis for a Metallodielectric Photonic Crystal}

In Sects. 6.2 and 6.3 we have dealt with dielectric photonic crystals. Photonic crystal structures can also be made of metals. But it is not easy to obtain negative refraction from the metallic photonic crystal structures since the EFCs 
of the metallic PCs are larger than the EFCs of air. Luo et al. theoretically demonstrated that it is indeed possible to obtain all-angle negative refraction by embedding metallic rods into a high-dielectric constant medium [44]. The main idea for using a medium with high dielectric permittivity is to increase the effective index of the photonic crystal. The advantage is that the EFCs will be lowered in frequency but the area occupied by EFCs in $k$ space will not change. Following this basic idea, we used a different approach for constructing the PC structure. Instead of embedding metallic rods into a high-dielectric medium, we combined dielectric rods and metallic rods together to form a metallodielectric PC. Metallodielectric PC could be considered as a metallic $\mathrm{PC}$ with a periodic dielectric perturbation. Positive dielectric constant is an attractive perturbation and causes lowering the frequency of the bands [50].

The metallodielectric photonic crystal is a square lattice of metallic and dielectric rods where the basis of the PC consists of a metallic and a dielectric rod placed along the diagonal of the square unit cell as given in the inset of Fig. 6.8b. Cylindrical alumina rods with a radius of $1.55 \mathrm{~mm}$ are used as the dielectric rods with $\varepsilon=9.61$. The metallic rods are made of aluminum and have a radius of $1.5 \mathrm{~mm}$. Both the metallic and dielectric rods have a height of $150 \mathrm{~mm}$.

We have computed the band diagrams of the metallic photonic crystal (Fig. 6.8a) and metallodielectric photonic crystal (Fig. 6.8b) for TM polarized EM waves. The radius of the dielectric rod is $0.136 a$, and the radius
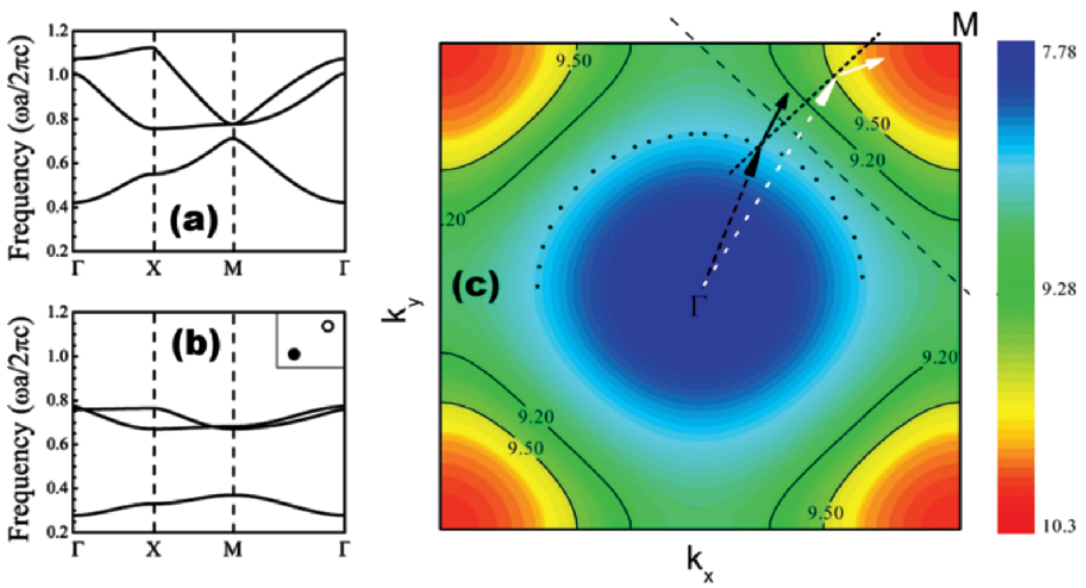

Fig. 6.8. (a) Calculated TM-polarized bands for the metallic photonic crystal, and (b) the metallodielectric crystal. (c) Equal-frequency contours (solid curves) are shown for the metallodielectric PC. Dotted circle is the free-space equal-frequency contour at $9.5 \mathrm{GHz}$. Free-space wave vector (black dashed arrow), free-space group velocity (black solid arrow), wave vector of the refracted waves in the $\mathrm{PC}$ (white dashed arrow), group velocity inside the PC (white solid arrow) are drawn 
of the metallic rod is $0.14 a$, where $a$ is the lattice constant and is equal to $11.0 \mathrm{~mm}$. Lattice constant is taken to be same in both structures. By comparing Fig. 6.8a and b we can conclude that the bands of the metallodielectric photonic crystal are lowered in frequency compared to the bands of the metallic photonic crystal.

Equal-frequency contours of the metallodielectric PC for TM polarization over the first Brillouin zone is plotted in Fig. 6.8c. The dotted circle is the EFC of air. The PC surface is aligned such that the normal vector to the air$\mathrm{PC}$ interface is along the $\Gamma M$ direction. Since the surface-parallel component of the wave vector is conserved, the wave vectors of the refracted beam can easily be obtained, as given in the figure. The group velocity of the incident waves and the group velocity of the transmitted waves fall on opposite sides of the surface normal. Therefore the incident waves are negatively refracted [34]. For the plotted EFCs, the magnitude of the largest surface-parallel wave vector component in air is smaller than the largest surface-parallel wave vector component in the photonic crystal. The range of incidence angles that are negatively refracted can be increased by lowering the bands without modifying the lattice parameters [50].

The electric field intensities are measured along the PC-air interface to demonstrate the negative refraction experimentally. The measurement method is same with the measurements indicated in the previous sections (Fig. 6.2a). Waves that are positively refracted are expected to emerge from the positive side of the surface, whereas negatively refracted waves are to emerge from the negative side. Measurement results for incidence angles of $\theta_{\mathrm{i}}=15^{\circ}, 25^{\circ}, 35^{\circ}$, and $45^{\circ}$ are provided in Fig. $6.9 \mathrm{a}-\mathrm{d}$, respectively. Between the frequencies 9.20 and $10.30 \mathrm{GHz}$ waves exit from the negative side of the PC meaning that $\mathrm{EM}$ waves are negatively refracted by the $\mathrm{PC}$ in this frequency range. Up to $9.20 \mathrm{GHz}$ the waves are positively refracted.

We have also performed FDTD simulations to see how the EM wave is refracted by the metallodielectric PC. We have sent an incident Gaussian beam to the air-PC interface and calculated electric field intensities inside and outside the $\mathrm{PC}$ at $9.70 \mathrm{GHz}$. The simulation results for two different incident angles $\theta_{\mathrm{i}}=15^{\circ}$ (Fig. 6.10a) and $\theta_{\mathrm{i}}=45^{\circ}$ (Fig. 6.10b) clearly show that the negative refraction takes place at this frequency. The effect of reflection is also clear in this picture. For higher incident angle the intensity (therefore the transmission) is lower due to the high reflections from the air- $\mathrm{PC}$ interface. Note that $9.70 \mathrm{GHz}$ is in the negative refraction frequency regime as calculated from the EFCs (Fig. 6.8c).

We have compared the experimental and theoretical results of the values of refractive indices at $\theta_{\mathrm{i}}=25^{\circ}$ incidence angle in Fig. 6.11. Between 9.00 and $9.19 \mathrm{GHz}$ the refractive indices are found to be positive. Around 9.20 GHz there is an abrupt change in the values and the sign of the index of refraction. In the close vicinity of $9.20 \mathrm{GHz}$ the indices of refraction are found to be high, +18 and -12 due to the flatness of EFS contours around $9.20 \mathrm{GHz}$ (Fig. 6.8c). The refractive indices are negative in the frequency range 

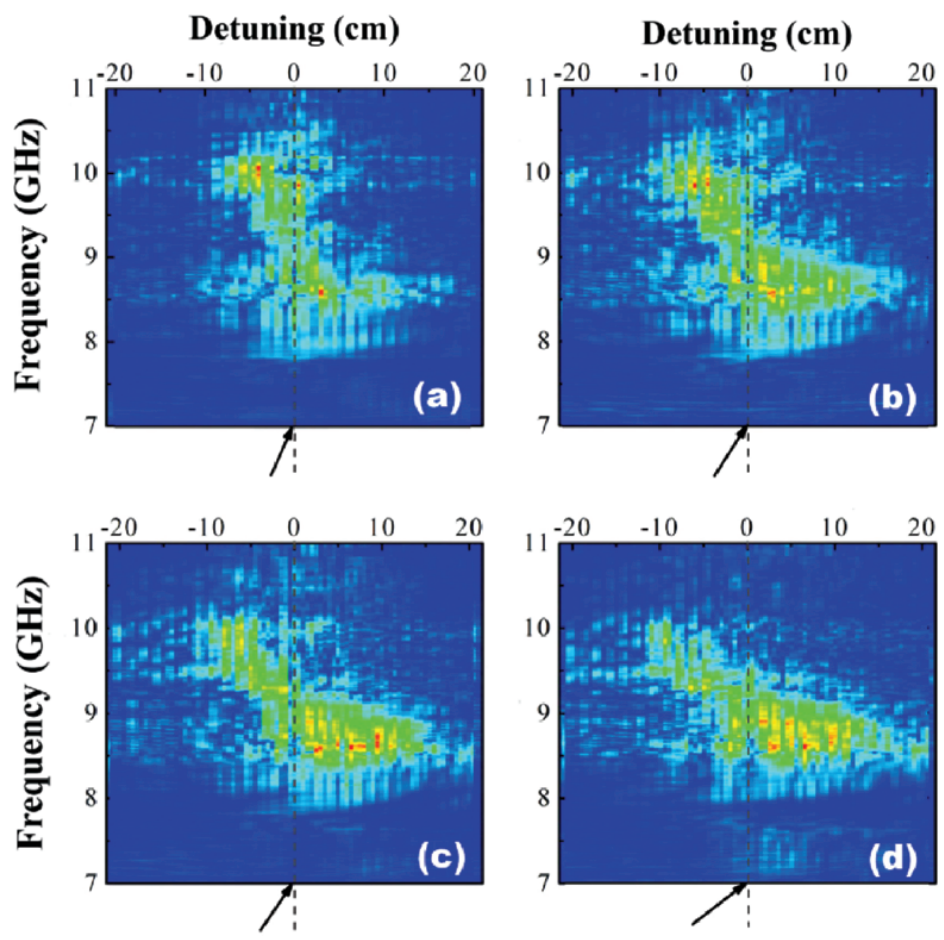

Fig. 6.9. Measured electric field intensities along the $\mathrm{PC}$-air interface for incident angles of (a) $\theta=15^{\circ}$, , b ) $\theta=25^{\circ}$, (c) $\theta=35^{\circ}$, and (d) $\theta=45^{\circ}$ for the metallodielectric PC. The intensities are plotted as a function of frequency. Up to $9.2 \mathrm{GHz}$ the structure is positively refracting and between 9.2 and $10.3 \mathrm{GHz}$ the structure refracts negatively
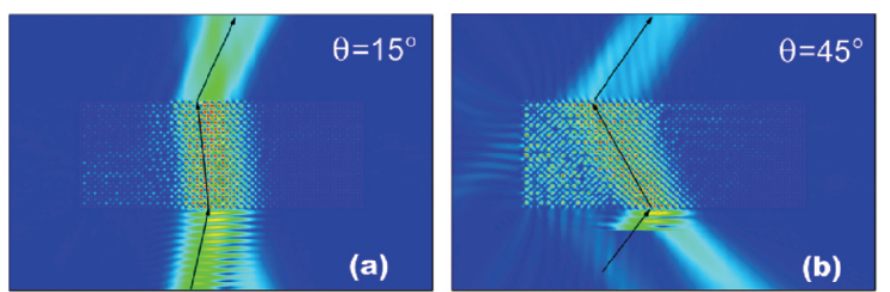

Fig. 6.10. Simulated electric field distributions for both angles of incidence. Incident angles of (a) $\theta=15^{\circ}$ and (b) $\theta=45^{\circ}$ for the metallodielectric PC. Negative refraction phenomenon is clearly observed for both angles of incidence

9.25-10.00 GHz. As seen in Fig. 6.11 the refractive indices depend strongly on the frequency. This is an expected behavior since the EFCs (Fig. 6.8c) are anisotropic throughout the frequency range of interest. Measured refractive index values are $-0.65,-0.85,-0.88$, and -0.96 for four different incidence 


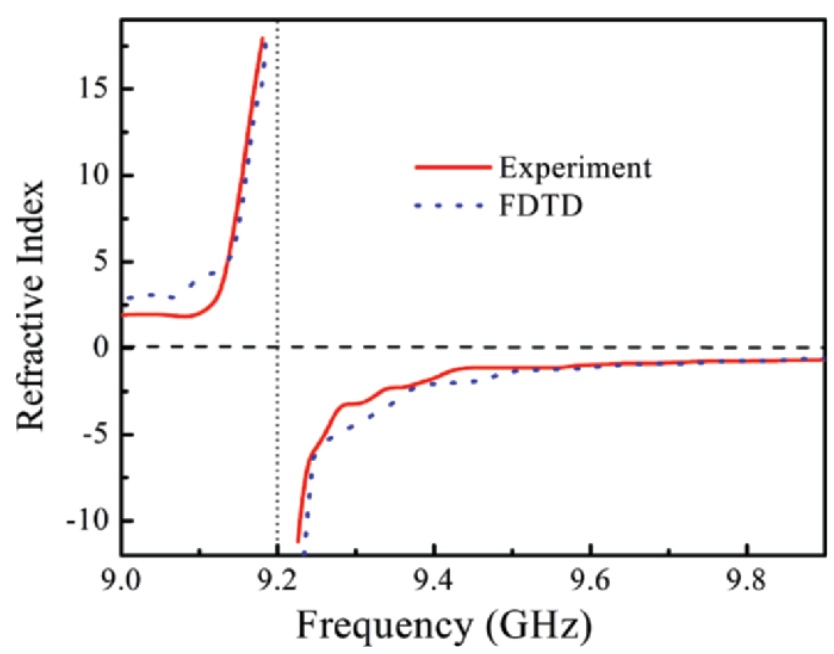

Fig. 6.11. Measured (solid line) and calculated (dashed line) refractive indices at an angle of incidence $15^{\circ}$ for the metallodielectric PC

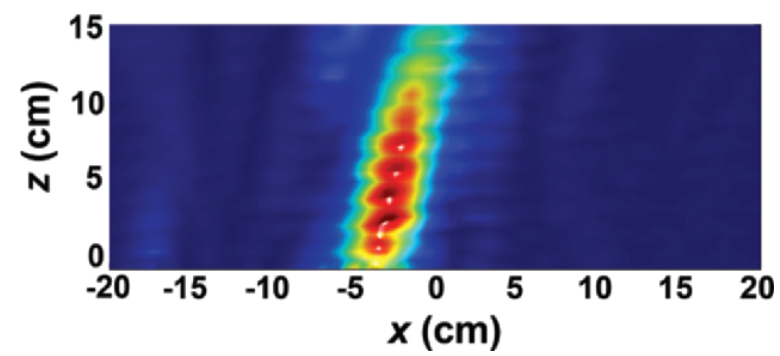

Fig. 6.12. Spatial intensity distribution of an outgoing EM wave at $3.70 \mathrm{GHz}$ along the $x-z$ plane of the metallodielectric PC. The wave is refracted negatively since the beam emerges from the negative side of the normal

angles of $15^{\circ}, 25^{\circ}, 35^{\circ}$, and $45^{\circ}$, respectively, at $9.70 \mathrm{GHz}$. These results clearly show that refractive index also depends on the incidence angle. To obtain uniform angle-independent negative indices of refraction two conditions must be satisfied. First, a circular EFC centered at the origin of the Brillouin zone is required. Second, the radius of the circular EFC must be decreasing with increasing frequency [50].

We have also scanned the intensity distribution of the EM wave by a monopole antenna mounted to a $2 \mathrm{D}$ scanning table with $\Delta x=\Delta z=2.5 \mathrm{~mm}$ steps. In our experiments we can only measure the power at a certain point, which corresponds to the time-averaged intensity at that point. Note that up to this point we have only scanned the field along PC-air interface for measuring the index of refraction. The resulting scanned field profile at $9.70 \mathrm{GHz}$ is plotted in Fig. 6.12. We can clearly see how the wave propagates after being refracted 


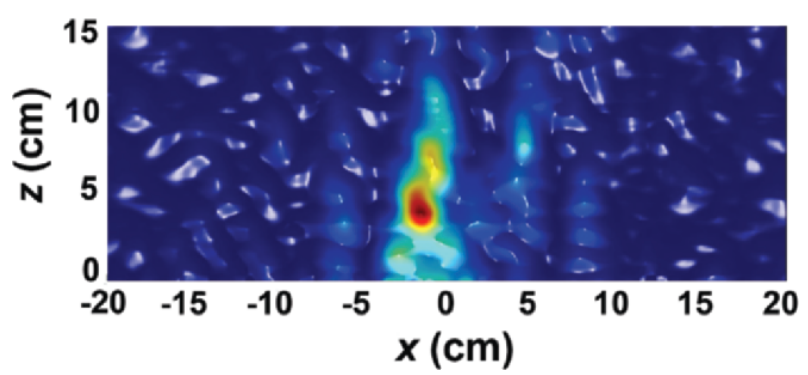

Fig. 6.13. Spatial intensity distribution of an omnidirectional source at $3.70 \mathrm{GHz}$ along the $x--z$ plane of the metallodielectric PC. Focusing through a slab of metallodielectric photonic crystal is observed

from the $\mathrm{PC}$-air interface. The intensities are normalized with respect to the maximum intensity. The incident EM wave has a Gaussian beam profile centered at $x=0$. Gaussian beam is shifted by $40 \mathrm{~mm}$ to the negative side of the normal as expected from a negatively refracting medium.

A similar field scan is performed for the observation of focusing through a PC slab lens. In this case we have used a monopole antenna as a point source to shine the air-PC interface (refer to Fig. 6.2b for setup). EM waves emerging from a point source located near a lens with negative refractive index will first be refracted through the first air-PC interface and will come into focus inside the PC. Then outgoing EM waves will face refraction again at the second PC-air interface and the refracted beam will meet the optical axis of flat lens, where the second focusing will occur. If the lens is not thick enough, the focusing may not occur inside the lens, which in turn will result in a diverging beam instead of a converging beam, even if the material is negatively refracting. Therefore, the thickness of the lens plays a crucial role for observing flat lens behavior.

Figure 6.13 provides the transmission spectrum for the omnidirectional source located at $d_{\mathrm{s}}=7 \mathrm{~cm}$ away from the PC lens. Number of layers along the propagation direction is $N_{\mathrm{z}}=10$. As seen in Fig. 6.13, an image is formed at a focal length of $z=4 \mathrm{~cm}$. Focusing is obtained both in the lateral and the propagation direction. If the slab was made of a positive refractive material, it would not be possible to observe a point focusing. For such positive refractive index slab lenses the beam will diverge as expected from ray optics. Therefore flat lens focusing is available only for negative refractive media.

\subsection{Conclusion}

In this chapter, the negative refraction and the focusing abilities of $2 \mathrm{D}$ dielectric and metallodielectric photonic crystals were investigated both experimentally and theoretically. We have observed that an effective index of refraction 
can be defined from the band structure of the PC, which, under convex EFCs, can take negative values and can be associated with refraction of EM waves through the PC. The isotropy and spectral range of the refractive indices depend strongly on the details of the band structure. The focusing abilities associated with negative refractive index are promising. We have observed that the subwavelength imaging and far-field focusing are achievable using $\mathrm{PC}$ with appropriate band structures. Our studies showed that negative refraction is available both for TM and TE polarized incident EM waves. Metallodielectric crystals are used to obtain negative refractive indices over a wide range of angles. Dielectric rods are inserted within metallic crystal, and the resulting band diagram is calculated and a lowering in frequency of the bands is observed.

The advantage of using photonic crystals as negative refractive media is that the transmission is higher compared to lossy LHM. Also the electromagnetic phenomena discussed here depend only on the refractive index of the dielectric material and on the geometrical parameters of the $2 \mathrm{D} \mathrm{PC}$, hence it is scalable across the electromagnetic spectrum. With advancing fabrication techniques, photonic crystals are now seen as essential building blocks of applications in the infrared and optical frequencies. It is much more difficult to scale the LHM that are made of metallic structures.

\section{Acknowledgments}

This work is supported by the European Union under the projects EU-DALHM, EU-NOE-METAMORPHOSE, EU-NOE-PHOREMOST, and TUBITAK under Project No. 104E090. Ekmel Ozbay acknowledges partial support from the Turkish Academy of Sciences.

\section{References}

1. V.G. Veselago, Sov. Phys. Usp. 10, 504 (1968)

2. D.R. Smith, W.J. Padilla, D.C. Vier, S.C. Nemat-Nasser, S. Schultz, Phys. Rev. Lett. 84, 4184 (2000)

3. R.A. Shelby, D.R. Smith, S.C. Nemat-Nasser, S. Schultz, Appl. Phys. Lett. 78, $489(2001)$

4. M. Bayindir, K. Aydin, P. Markos, C.M. Soukoulis, E. Ozbay, Appl. Phys. Lett. 81, 120 (2002)

5. K. Aydin, K. Guven, M. Kafesaki, L. Zhang, C.M. Soukoulis, E. Ozbay, Opt. Lett. 29, 2623 (2004)

6. R.A. Shelby, D.R. Smith, S. Schultz, Science 292, 77 (2001)

7. C.G. Parazzoli, R.B. Greegor, K. Li, B.E. Koltenbah, M. Tanielian, Phys. Rev. Lett. 90, 107401 (2003)

8. A.A. Houck, J.B. Brock, I.L. Chuang, Phys. Rev. Lett. 90, 137401 (2003)

9. K. Aydin, K. Guven, C.M. Soukoulis, E. Ozbay, Appl. Phys. Lett. 86, 124102 (2005) 
10. K. Aydin, I. Bulu, E. Ozbay, Opt. Express 13, 8753 (2005)

11. J.B. Pendry, A.J. Holden, D.J. Robbins, W.J. Stewart, J. Phys. Condens. Matter 10, 4785 (1998)

12. J.B. Pendry, A.J. Holden, D.J. Robbins, W.J. Stewart, IEEE Trans. Microw. Theory Tech. 47, 2075 (1999)

13. J.B. Pendry, Phys. Rev. Lett. 85, 3966 (2000)

14. E. Yablonovitch, Phys. Rev. Lett. 58, 2059 (1987)

15. S. John, Phys. Rev. Lett. 58, 2486 (1987)

16. J.D. Joannopoulos, R.D. Meade, J.N. Winn, Photonic Crystals: Molding the Flow of Light (Princeton University Press, Princeton, NJ, 1995)

17. C.M. Soukoulis (ed.), Photonic Crystals and Light Localization in the 21st Century (Kluwer, Norwell, MA, 2001)

18. K. Sakoda, Optical Properties of Photonic Crystals (Springer, Berlin Heidelberg New York, 2001)

19. E. Ozbay, G. Tuttle, R. Biswas, K.M. Ho, J. Bostak, D.M. Bloom, Appl. Phys. Lett. 65, 1617 (1994)

20. E. Ozbay, B. Temelkuran, Appl. Phys. Lett. 69, 743 (1996)

21. A. Mekis, J.C. Chen, I. Kurland, S. Fan, P.R. Villeneuve, and J.D. Joannapoulos, Phys. Rev. Lett. 77, 3787 (1996)

22. P.R. Villeneuve, D.S. Abrams, S. Fan, J.D. Joannopoulos, Opt. Lett. 21, 2017 (1996)

23. B. Temelkuran, E. Ozbay, J.P. Kavanaugh, G. Tuttle, K.M. Ho, Appl. Phys. Lett. 72, 2376 (1998)

24. H. Kosaka, T. Kawashima, A. Tomita, M. Notomi, T. Tamamura, T. Sato, S. Kawakamib, Appl. Phys. Lett. 74, 1370 (1999)

25. M. Bayindir, E. Ozbay, Phys Rev. B 62, R2247 (2000)

26. M. Loncar, D. Nedeljkovic, T. Doll, J. Vuckovic, A. Scherer, T.P. Pearsall, Appl. Phys. Lett. 77, 1937 (2000)

27. S. Noda, M. Yokoyama, M. Imada, A. Chutinan, M. Mochizuki, Science 293, $1123(2001)$

28. M. Bayindir, S. Tanriseven, E. Ozbay, Appl. Phys. A-Mater. Sci. Process. 72, $117(2001)$

29. E. Ozbay, M. Bayindir, I. Bulu, E. Cubukcu, IEEE J. Quantum Electron. 38, $837(2002)$

30. S. Enoch, B. Gralak, G. Tayeb, Appl. Phys. Lett. 81, 1588 (2002)

31. M.F. Yanik, S. Fan, Phys. Rev. Lett. 92, 083901 (2004)

32. B. Gralak, S. Enoch, G. Tayeb, J. Opt. Soc. Am. A 17, 1012 (2000)

33. M. Notomi, Phys. Rev. B 62, 10696 (2000)

34. C. Luo, S.G. Johnson, J.D. Joannopoulos, J.B. Pendry, Phys. Rev. B 65, 201104 (2002)

35. S. Foteinopoulou, E.N. Economou, C.M. Soukoulis, Phys. Rev. Lett. 90, 107402 (2003)

36. E. Cubukcu, K. Aydin, E. Ozbay, S. Foteinopoulou, C.M. Soukoulis, Nature 423, 604 (2003)

37. P.V. Parimi, W.T. Lu, P. Vodo, J. Sokoloff, J.S. Derov, S. Sridhar, Phys. Rev. Lett. 92, $127401(2004)$

38. A. Berrier, M. Mulot, M. Swillo, M. Qiu, L. Thylén, A. Talneau, S. Anand, Phys. Rev. Lett. 93, 073902 (2004)

39. H. Kosaka, T. Kawashima, A. Tomita, M. Notomi, T. Tamamura, T. Sato, S. Kawakami, Phys. Rev. B 58, R10096 (1998) 
40. S. Foteinopoulou, C.M. Soukoulis, Phys. Rev. B 67, 235107 (2003)

41. E. Cubukcu, K. Aydin, E. Ozbay, S. Foteinopoulou, C.M. Soukoulis, Phys. Rev. Lett. 91, 207401 (2003)

42. P.V. Parimi, W.T. Lu, P. Vodo, S. Sridhar, Nature 426, 404 (2003)

43. C. Luo, S.G. Johnson, J.D. Joannopoulos, J.B. Pendry, Phys. Rev. B 68, 045115 (2003)

44. C. Luo, S.G. Johnson, J.D. Joannopoulos, J.B. Pendry, Opt. Express 11, 746 (2003)

45. S. Foteinopoulou, C.M. Soukoulis, Phys. Rev. B 72, 165112 (2005)

46. R. Moussa, S. Foteinopoulou, L. Zhang, G. Tuttle, K. Guven, E. Ozbay, C.M. Soukoulis, Phys. Rev. B 71, 085106 (2005)

47. K. Guven, K. Aydin, K.B. Alici, C.M. Soukoulis, E. Ozbay, Phys. Rev. B 70, 205125 (2004)

48. E. Ozbay, K. Guven, E. Cubukcu, K. Aydin, B.K. Alici, Mod. Phys. Lett. B 18, $1275(2004)$

49. A. Martínez, H. Míguez, A. Griol, J. Martí, Phys. Rev. B 69, 165119 (2004)

50. I. Bulu, H. Caglayan, E. Ozbay, Phys. Rev. B 72, 045125 (2005)

51. P. Vodo, P.V. Parimi, W.T. Lu, S. Sridhar, R. Wing, Appl. Phys. Lett. 85, 1858 (2005)

52. P. Vodo, P.V. Parimi, W.T. Lu, S. Sridhar, Appl. Phys. Lett. 86, 201108 (2005) 